\title{
2
}

\section{Establishing an academic domain}

\author{
Hiroyuki Yoshikawa \\ Professor Emeritus and Past President of the University of Tokyo \\ President of Science Council of Japan \\ President of Japan Society for the Promotion of Science \\ Science Advisor to the Minister
}

\section{1-14-13-918 Tsukijima, Chuo-ku, Tokyo 104, JAPAN \\ Tel: +81-3-3532-5558 Fax: +81-3-3532-5558}

Any sphere of scholarship is made in the following ways:

(1) Limitations are put on the subjects to be treated by that realm of scholarship. Thus, the assemblage of subjects is fixed, and this is called collection.

(2) Simple, fundamental principles are brought out from the relationship among elements of the assemblage of selected subjects. These are called laws.

(3) A system concerned with phenomena that arise not in contradiction with the laws is described. This is the theoretic system.

Probably such theoretic systems comprise the major part of many academic spheres. They are normally arranged in a form convenient for learning, teaching, as well as application.

Now since this alone is both too simple and yet difficult to comprehend, let us consider the example of Newton's dynamics. As Newton in actuality presented the world with the entirety of his theory of dynamics in his Principia-a Mathematical Theory of Natural Philosophy, which is believed to have been published in 1687, this means he was 45 years old at the time. In view of the fact that his interest in dynamics sprouted during his student years, this calculates to completion of the theory taking more than twenty years.

Moreover, since Kepler's theory concerning the movements of celestial bodies already existed at the time, and researchers of those days also continued to undertake various studies, getting to the process behind the materialization of Newton's dynamics is extremely complicated. Yet what we want to know here is not the historical evidence concerning the materialization of his dynamics but the theoretical structure lying in the background of that process.

The first topic is collection. Newton is known to have been interested in several phenomena when he was a student. Issues like the real nature of matter, place, time, movement, the order of the universe, energy, light, color, the sense of sight, sensation, and so on cover the broad range of subjects of contemporary scholarship that also treats physical laws, chemistry, organisms, human beings, etc. 
From the standpoint of traditional natural philosophy, the disposition referred to as overall explication is the value of the philosophical theory, so that all recognizable subjects must be listed for the time being as the starting point. In other words, the inclusion of all subjects can be said to be a requirement of collection.

This is strange when regarded from modern scholarship. Or else it can be called reckless, because contemporary scholarship is divided into many realms. In addition, the ambition to make thorough, coordinated explanations of all subjects is never brought forth but, instead, gets ensconced in explications of intently and modestly limited subjects.

While Newton started off by standing in a classical perspective and having interest in all subjects, he nevertheless proceeded to limit his topics and eventually pared them down just to moving bodies as dynamic actors. These moving bodies did not consist solely of celestial ones but also included the apples on Earth. When considered now, that seems only natural, but it must have made for an extremely böld collection in Newton's days.

Newton successively put limitations on these sorts of subjects. He also attempted to give an as coordinated explanation as possible of each one. Light and chemical phenomena are included among them. Probably his plan was not to try to explain all the subjects that interested him as a natural philosopher but rather to create a collection by cutting off particular topics from all others through clear elucidation of the perspective and to make an explanation peculiar to handling that alone. Then, he stood in the position of reaching an understanding of all subjects by amassing a comprehension of each collection, scattered though they were.

Here doubts arise as to why Newton made a collection that included both celestial bodies and apples while he eliminated light from it. Expressed another way, this means to ask on what basis did he fix his perspective and then build his collection.

An important characteristic of what we know as the modern academic system is its division into several spheres. However, considering that the basis for and necessity of those divisions can hardly be explained, the aforementioned doubts should be viewed with grave importance in present times, too.

It is certain that Principia would not have seen completion if moving bodies and light had been put into the same collection. Nevertheless, there are no grounds for making the explanation that Newton fixed his viewpoint on moving bodies and limited the subjects in order to bring his dynamics to completion. That essentially does not result in answering doubts, because the logical relationship is that only after there are limited subjects does it become possible for systems of explanation to be brought out. Besides, a system of explication could not possibly exist without limitations on the subject.

The realms that characterize modern scholastic systems were essentially initiated by Newton. The fact that the structure of the realm known as the dynamics of moving objects became the true standard for scholarship thereafter attests to Newton's boundless greatness. Since how he made that realm-in other words, that collection-can not be explained, we can only say that it owes its materialization to his greatness. 
The next step in making scholarship is to bring out laws. When a collection is given, various amounts related to it are observed, and the relationship among the various amounts can then be obtained as a result of the observations. The collection under Newton's dynamics is of moving objects; things like density, capacity, mass, volume of movement, and strength are abstracted as amounts in relation to that. Brought out next are the fundamental principles - in other words, laws necessary and adequate for recognizing the feasibility of all movements that have actually been observed, including those of celestial bodies and falling apples.

Laws in the case of Newton's dynamics mean the following three concerned with the uniform motion of bodies to which no strength has been added, the changes in the amount of motion of bodies to which power has been added, and action/reaction. The framework of Newton's dynamics says that those three laws can be employed to explain all movement observed, including both the movement of bodies for which the influence of their viscosity on earth has been considered, too, and the movement of celestial bodies.

There is extraordinary potency in asserting that every kind of movement can be explained with just three items. Thereupon arises the question concerning how Newton discovered such powerful laws. Yet it is also difficult to respond to that. There are no such explanations in Principia, and it is said that perhaps Newton himself could not explain it.

Nevertheless, the extraction of these laws is believed to have played a leading role from modern times on at least in the progress of the natural sciences. A detailed examination of that was conducted by Charles Sanders Peirce.

When Newton established his three laws, they perhaps ought to have been called a hypothesis. This is because the laws can be deemed correct once all movements inferred as lying within the realm of possibility according to these three completely match all movements observed in reality. If things inferred as likely to occur do not actually happen, or if movements observed in reality could not have been inferred under the laws, then those laws should not be regarded as correct.

Consequently, since a law has not undergone sufficient investigation at the time of its establishment, it should be considered a hypothesis at first. Therefore, the extraction of a law can be called the formation of a hypothesis. However, Peirce called this influence abduction. The original meaning of that word usually refers to kidnaping, and it would certainly not be wrong to say that the term gives off a sense of taking something by force and without good reason.

I have emphasized that scholarship is also something thought of by human beings, and in this case what is known as inference has played the leading part. If we divide that very broadly and generally, we get deductive inference, inductive inference, and formation of a hypothesis. If we think in terms of syllogism, inference in the relationship among rules, precedents, and results can be classified in the following ways:

Deductive inference: inferring the results from rules and precedents Inductive inference: inferring rules from precedents and results Formation of hypothesis: inferring precedents from rules and results. 
This can be easily understood instinctively if we consider this in terms of the following examples provided by Peirce:

Rule: All the beans in this bag are white;

Precedent: These beans were in this bag;

Result: These beans are white,

Here there is no worry about mistakes occurring with deduction.

Actually under Newton's theory rules are law; precedents are included in the collection of celestial bodies and apples, and results in the assemblage of movements that could possibly happen. If one precedent is selected, then a single movement corresponding to that will get designated. When possible, it is acceptable to put those designations on a correspondence chart that includes everything; but in reality they have come to be calculated through mathematical solutions. The correspondence can not be indicated on a chart if it becomes infinite. Thus, this calculation, which is far more rational than the chart, will perform a valuable job.

In any case, we can understand that Newton's theoretic system of dynamics is deductive. If we take a look at Principia, the book that published his dynamics, only two pages are devoted to laws, while results for each precedent are indicated in the several hundred pages remaining. The book consists almost entirely of deduction.

Well, then how were the laws, which are most important in the materialization of this great book, brought out? I earlier wrote that even Newton himself did not know, but the materializing process took long years and must have been complicated. Yet if we consider that as a question of inference, which have already been classified, it will end up as induction. It is easy to infer after taking some beans out of a bag and seeing they are all while that every bean in the bag is white. It is not something a person spends grueling years pondering over. However, to make strict distinctions between induction and the formation of hypothesis is not simple, and there are times when we can not say that the extraction of laws is induction.

In reference to this, Peirce clearly related the difference between induction and hypothesis-forming. He, thus, wrote an explanation to the effect that in contrast to the essence of induction, which involves inferring a similar fact from another fact, the formation of a hypothesis lies in inferring from a certain fact a different kind of fact which is not directly observed.

Precedent is generally dispersed. Consequently, because there are many cases in which inference for it is inference for a different type of fact, it is easy to explain inference of a precedent as formation of a hypothesis. In the case of beans, the rule is that all are white. Thus, the upshot is that when some red beans get mixed in, they will be said to come from a different bag. Therefore, the need arises here to bring in the new thinking known as a different bag. However, with induction, if some red beans get mixed in with what are supposed to be all white, we only have to revise the rule a little bit.

In the world of scholarship, proposals for completely new laws-rather than slight revisions in the laws- tend to be highly evaluated as new theories. While Peirce sug- 
gested that various types of cases occur continuously in between slight revisions in laws and new proposals, he regarded proposals for laws in a new theory as a supplementary mixture of induction and formation of a hypothesis. He also claimed that the formation of a hypothesis - that is to say, abduction - has played an important role in the rapid progress of the scholarship known as new theories.

Peirce, furthermore, gave deep thought to abduction. However, in the end he was unable to explain that action clearly and concluded that it is a strange human capacity. Accordingly, his ideas conclude that Newton splendidly extracted forceful laws because he was great. Here, too, Newton's greatness comes through.

Now let us consider abduction a bit closer to ourselves. Speaking in terms of our daily lives, it is conception; or in words popular nowadays, it is an act demanding originality. I will next give some original examples.

As a rule, we can say that "human beings will die." From the precedent that "Socrates is a human being," it can be inferred that "Socrates is likely to die." That is deduction. In this style, under the rule "human beings will die," we are to have witnessed something dying before our eyes. This is a result.

However, when we have no knowledge about the thing that died-in other words, when we do not know what the precedent is-, we infer by developing a hypothesis. To conclude that the object is a person because it died is a frank and perhaps original inference. When it becomes clear in a later age that what died was really a cockroach, this will mean the absurd, nonsensical inference that "a cockroach is a human being" was made at the time.

Nevertheless, the essence of abduction can be found here. Abduction increases knowledge for people in the intrinsic sense, but the possibility of erring is present as well. In contrast to deduction, where mistakes are not made, and induction, in which it is possible to correct an error already made, when a slip occurs with abduction, the next inference will begin all over from zero.

\section{BIOGRAPHY}

Hiroyuki Yoshikawa received Bachelor of Engineering and Doctor of Engineering from the University of Tokyo in 1956 and 1963, respectively. He was a professor of the University of Tokyo from 1966 to 1997 where he was the President from 1993 to 1997. He has been a Science Advisor to the Minister since April 1997, the President of Science Council of Japan since July 1997, and the President of Japan Society for the Promotion of Science since September 1997. His research area covers General Design Theory, Intelligent CAD, Theory of Reliability and Maintenance, and Maintenance Robot. He is a member of IFIP W.G5.7 and 5.2. He is the chairman of the Committee of Intelligent Manufacturing Systems Promotion, Ministry of International Trade \& Industry, the chairman of the Committee of Study, Japan Committee of Industrial Performance, and the Deputy President of the Committee of Science \& Technology Policy (OECD). He is the past president of International Institution of Production Engineering Research and Japan Society of Precision Engineering. 